\title{
Post-operative pain management modalities employed in clinical trials for adult patients in LMIC; a systematic review
}

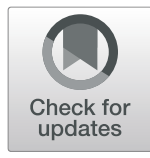

Gauhar Afshan 1* (D), Robyna Irshad Khan', Aliya Ahmed', Ali Sarfraz Siddiqui², Azhar Rehman², Syed Amir Raza², Rozina Kerai ${ }^{1}$ and Khawaja Mustafa ${ }^{1}$

\begin{abstract}
Background: Unrelieved postoperative pain afflicts millions each year in low and middle income countries (LMIC). Despite substantial advances in the study of pain, this area remains neglected. Current systematic review was designed to ascertain the types of clinical trials conducted in LMIC on postoperative pain management modalities over the last decade.

Methods: A comprehensive search was performed in June 2019 on PubMed, Cochrane Library, CINAHL Plus, and Web of Science databases to identify relevant trials on the management of postoperative pain in LMIC. Out of 1450 RCTs, 108 studies were reviewed for quality evidence using structured form of critical appraisal skill program. Total of 51 clinical trials were included after applying inclusion/exclusion criteria.

Results: Results are charted according to the type of surgery. Eleven trials on laparoscopic cholecystectomy used multimodal analgesia including some form of regional analgesia. Different analgesic modalities were studied in 4 trials on thoracotomy, but none used multimodal approach. In 11 trials on laparotomy, multimodal analgesia was employed along with the studied modalities. In 2 trials on hysterectomy, preemptive pregabalin or gabapentin were used for reduction in rescue analgesia. In 13 trials on breast surgical procedures and 10 on orthopaedic surgery, multimodal analgesia was used with some form of regional analgesia.

Conclusion: We found that over the past 10 years, clinical trials for postoperative pain modalities have evolved in LMIC according to the current postoperative pain management guidelines i.e. multi-modal approach with some form of regional analgesia. The current review shows that clinical trials were conducted using multimodal analgesia including but not limited to some form of regional analgesia for postoperative pain in LMIC however this research snapshot (of only three countries) may not exactly reflect the clinical practices in all 47 countries.

Post Operative Pain Management Modalities Employed in Clinical Trials for Adult Patients in LMIC; A Systematic Review.
\end{abstract}

Keywords: Post-operative pain, LMIC, Multimodal analgesia

\footnotetext{
* Correspondence: gauhar.afshan@aku.edu

'Department of Anaesthesiology, 2nd floor Private Wing, Aga Khan University, P.O. Box 3500, Stadium Road, Karachi 74800, Pakistan

Full list of author information is available at the end of the article
}

(c) The Author(s). 2021 Open Access This article is licensed under a Creative Commons Attribution 4.0 International License, which permits use, sharing, adaptation, distribution and reproduction in any medium or format, as long as you give appropriate credit to the original author(s) and the source, provide a link to the Creative Commons licence, and indicate if changes were made. The images or other third party material in this article are included in the article's Creative Commons licence, unless indicated otherwise in a credit line to the material. If material is not included in the article's Creative Commons licence and your intended use is not permitted by statutory regulation or exceeds the permitted use, you will need to obtain permission directly from the copyright holder. To view a copy of this licence, visit http://creativecommons.org/licenses/by/4.0/ The Creative Commons Public Domain Dedication waiver (http://creativecommons.org/publicdomain/zero/1.0/) applies to the data made available in this article, unless otherwise stated in a credit line to the data. 


\section{Background}

"Despite substantial advances in pain research in recent decades, inadequate acute pain control is still more the rule than the exception," concluded international association for study of pain (IASP) while observing global year against acute pain in 2010-2011. Available data shows a large burden of acute pain in the developed countries, inferring logically, this burden is significantly higher in low and middle-income countries (LMIC). Anaesthesia and related specialties have been reporting the enormity of the burden of pain and suffering in LMIC citing disproportionately limited resources, lack of regulations, and paucity of pain education as the main reasons [1].

Causes of acute pain are numerous, including but not limited to, trauma, burn injury, medical illness, labour, violence, war, man-made and natural disasters, road traffic accidents, and post-operative pain; some being more prevalent in LMIC. Political and social instability in these countries compound the crisis and multiply the acute pain burden manifold [2]. Reported statistics list post-operative pain as the most predominant type of acute pain in LMIC. Absence of efficient basic health care, lack of preventive health, and non-existent disease screening leads to patients presenting with advanced pathology that requires extensive surgical procedures and hence more severe pain [3, 4]. Another reason of poor postoperative pain management is a dearth of strong opioids. Measured in terms of distribution of opioids, only 0.1 metric ton was distributed to LMIC out of a total of 298.5 metric tons of morphine distributed in 2010-2013 in the entire world [5].

Effective postoperative pain management is unquestionably a basic human right. The importance of effective pain relief has long been realized and acute pain services (APS) are operational in majority of the hospitals in the developed world for decades. Big data is available on the subject of postoperative pain management with resultant comprehensive guidelines for the assistance of anaesthesiologists and other physicians managing pain [6-8]. The panel constituted to review literature and formulate acute postoperative pain management guidelines for American Pain Society, American Society for Regional Anesthesia, and American Society of Anesthesiologists (2016) observed that the evidence supports use of multimodal analgesia in most situations though the exact components of multimodal regimen would differ depending upon the patient, setting, and surgical procedure [8]. These guidelines, though quite practical, may not be applicable in their entirety to all health care facilities in the LMIC.

In this age of electronic media, anaesthesiologists, surgeons, and allied health care providers of LMIC are well informed about current recommendations and guidelines but they are hindered by limitation of resources and other factors. Most research, currently available in PubMed, Google and other common search engines, has been conducted in developed countries and their findings might not be acceptable across the world so it is essential to review the published research from LMIC. Current systematic review was designed to chronicle the types of post operative pain management modalities employed in clinical trials for adult patients in LMIC over the last decade.

\section{Methods}

\section{Search strategy}

A systematic literature search was conducted with the assistance of a librarian in PubMed, Cochrane Library, CINAHL Plus, and Web of Science databases to identify all relevant studies on the management of postoperative pain in LMIC. A comprehensive search strategy was developed using a combination of $\mathrm{MeSH}$ term "pain, postoperative" with keywords "postoperative pain", "postoperative pain management", "postoperative pain relief", "postoperative analgesia", "postoperative surgical pain" with suitable Boolean searching $[9,10]$. We used the list of LMICs generated by the World Bank which includes 47 countries with a gross national income (GNI) per capita between $\$ 1026$ and $\$ 3995$. We included all 47 countries as per the list in our Boolean search [11].

A filter was applied for limiting the search to only human studies published from January 2008 to - June 2019. A total of 2885 studies were extracted and after removing duplicates, 2196 studies were selected. A total of 1450 randomized control trials (RCTs) were found out of 2196 in the pre-specified list of 47 countries.

\section{Searching and data abstraction}

Systematic review team comprised of five anaesthesiologists, one nurse, one biostatistician and one librarian. Two authors in each pair (total of three pairs of five anesthesiologists and one nurse) independently reviewed all potentially eligible 1450 RCTs. A total of 1342 were excluded after screening titles, reviewing abstracts and considering the objectives.

Full-text versions of 108 RCTs were reviewed using 11 questions, based on the structured form of CASP (critical appraisal skill program) by the same reviewers. Disagreements were resolved through open discussion and consensus. Finally, inclusion/exclusion criteria were applied. Common surgical procedures i.e. laparoscopic cholecystectomy, mastectomy, total abdominal hysterectomy, laparotomy, and orthopedic extremity surgery were included. Studies were excluded if post- operative pain management was provided for less than $24 \mathrm{~h}$ and/or no rescue analgesia was planned. Finally we selected 
RCTs fulfilling the inclusion \& exclusion criteria for the review as mentioned in the PRISMA diagram (Fig. 1). Patient characteristics (age and gender), study characteristics (name of the country and type of surgery), information on pain severity, pain measurement scale, types of different pain modalities (used), rescue analgesia (used), and duration of postoperative pain control were recorded in a structured format (Table 1). Systematic review team ensured that important studies were not missed; however publication bias is a possibility despite the due diligence observed while conducting the literature search.

\section{Results}

A total of 51 RCTs were included for the review. It is worth noting that only three countries among the list of LMIC have published RCTs fulfilling the predetermined

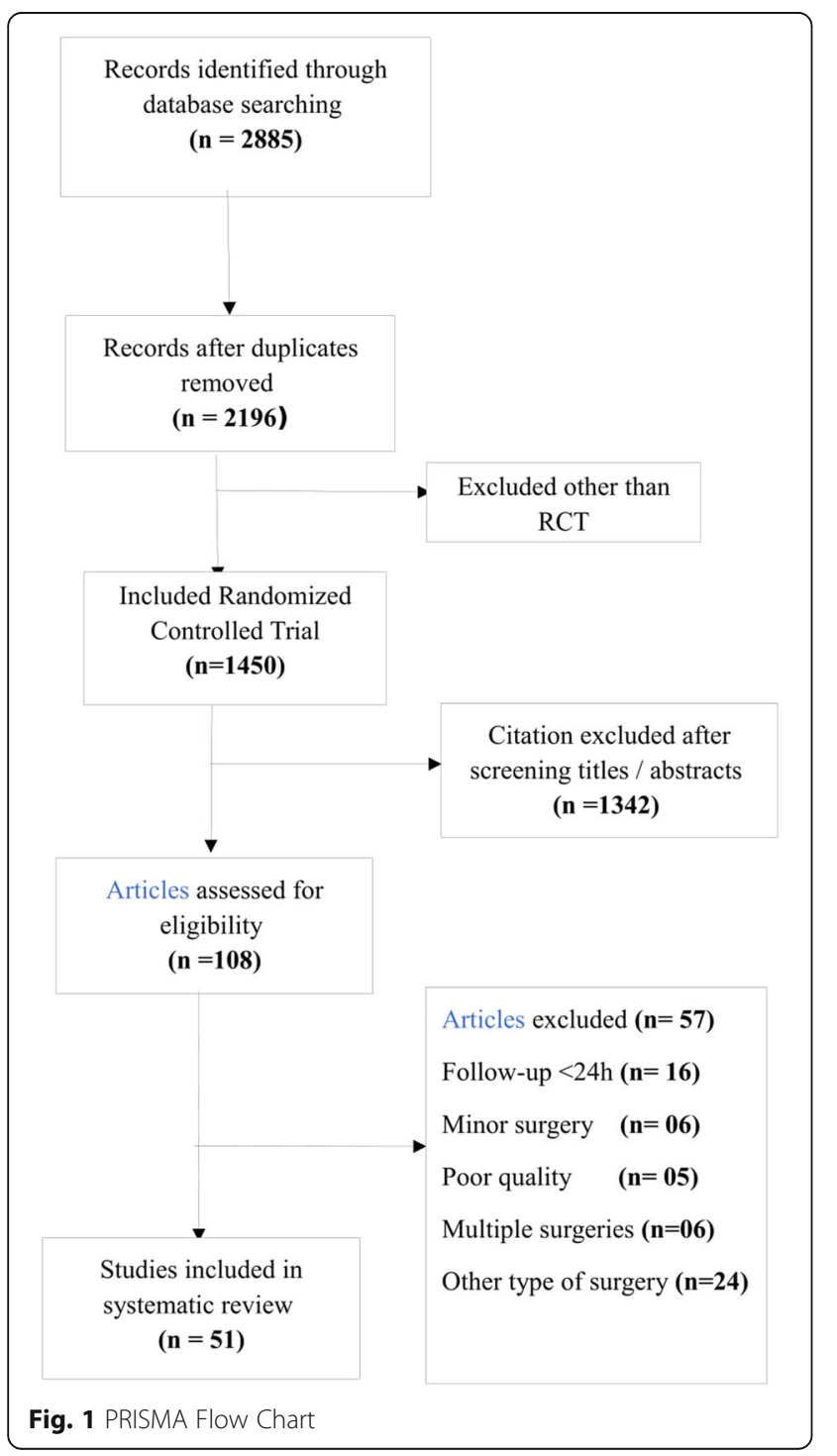

inclusion \& exclusion criteria. The review results were charted according to the type of surgery.

\section{Laparoscopic cholecystectomy}

Total of 11 RCTs [12-22] were included in the review (Table 1). These studies collectively described 842 patients of both genders with age range of 18-70 years. Majority used some form of regional analgesia. Transversus abdominis Plane (TAP) block comparing conventional and subcostal approaches was used in two RCTs [12, 21], and TAP block comparing two local anesthetics (LA) in one [14]. TAP block was compared with LA infiltration of incisional wounds in one RCT [18]. Intraperitoneal infiltration of LA comparing different drugs was used in two trials $[13,15]$ while intraperitoneal LA infiltration was compared with placebo in one [22]. One study compared LA infiltration of incisional wounds with abdominal plane blocks [16]. Oral Pregabalin was compared with Celecoxib in one trial [17], oral Pregabalin with Alprazolam in one [19] and Gabapentine with placebo in one [20]. All trials used multimodal analgesia for pain management, while comparing one or more modalities.

\section{Thoracotomy}

Total of 4 [23-26] RCTs were included for postoperative pain management following thoracotomies (Table 1). These trials collectively described 170 patients of both genders with age range of 18-70 years [23-26]. Various analgesic modalities have been studied including continuous thoracic epidural analgesia, serratus anterior plane block (SAPB), and continuous paravertebral block. None of the trials used multimodal approach. Three RCTs studied regional blocks with rescue analgesia [24-26] while one RCT studied continuous thoracic epidural analgesia without rescue analgesia [23]. Continuous Paravertebral dexmedetomidine was also used in one trial to decrease the intraoperative anaesthetic requirement and postthoracotomy pain syndrome [25].

\section{Laparotomy / Other Abdominal Surgery}

A total of 11 RCTs [27-37] were identified related to laparotomies and other open abdominal surgeries including a total number of 869 patients aged between 18 and 75 years (Table 1). Nine trials included patients of both genders, while two included females only. Various analgesic modalities were studied for postoperative pain relief following laparotomies and open abdominal procedures. One trial employed rectus sheath block comparing $0.25 \%$ bupivacaine with saline [27]. Another trial studied continuous wound infusion comparing bupivacaine with saline [28]. Thoracic epidural was used in five 
Table 1 Characteristics of the published randomized controlled trails presented with respect to type of surgery

\begin{tabular}{|c|c|c|c|c|c|c|c|c|c|c|c|}
\hline Authors & Country & $\begin{array}{l}\text { Age } \\
\text { Range }\end{array}$ & Gender & $\mathbf{N}$ & Groups & $\mathbf{N}$ & Modalities Studied & $\begin{array}{l}\text { Pain } \\
\text { Assessment } \\
\text { Tool }\end{array}$ & $\begin{array}{l}\text { Modalities } \\
\text { used }\end{array}$ & $\begin{array}{l}\text { Duration } \\
\text { of post- } \\
\text { operative } \\
\text { Pain } \\
\text { studied }\end{array}$ & $\begin{array}{l}\text { Rescue } \\
\text { Analgesia }\end{array}$ \\
\hline \multirow[t]{3}{*}{$\begin{array}{l}\text { Bhatia et al., } \\
\text { (2014) [12] }\end{array}$} & India & $18-60$ & Both & 60 & 3 & 20 & $\begin{array}{l}\text { Ultrasound-guided posterior } \\
\text { transversus abdominis plane } \\
\text { (TAP) block with } 15 \mathrm{~mL} \text { of } \\
0.375 \% \text { ropivacaine }\end{array}$ & $\begin{array}{l}\text { VAS } \\
\text { (Visual } \\
\text { Analogue } \\
\text { Scale) }\end{array}$ & Single & $24 \mathrm{~h}$ & Tramadol \\
\hline & & & & & & 20 & $\begin{array}{l}\text { Ultrasound-guided subcostal } \\
\text { TAP block with } 15 \mathrm{~mL} \text { of } \\
0.375 \% \text { ropivacaine }\end{array}$ & & & & \\
\hline & & & & & & 20 & Control & & & & \\
\hline \multirow[t]{3}{*}{$\begin{array}{l}\text { Shukla et al., } \\
\text { (2015) [13] }\end{array}$} & India & $18-60$ & Both & 120 & 3 & 40 & $\begin{array}{l}\text { Intraperitoneal bupivacaine } \\
50 \mathrm{ml} 0.25 \%+5 \mathrm{ml} \text { Normal } \\
\text { saline }\end{array}$ & VAS & Single & $24 \mathrm{~h}$ & Diclofenac \\
\hline & & & & & & 40 & $\begin{array}{l}\text { Bupivacaine } 50 \mathrm{ml} \mathrm{0.25 \% +} \\
\text { tramadol } 1 \mathrm{mg} / \mathrm{kg} \text { (diluted in } \\
5 \mathrm{ml} \mathrm{NS} \text { ) }\end{array}$ & & & & \\
\hline & & & & & & 40 & $\begin{array}{l}\text { Bupivacaine } 50 \mathrm{ml} 0.25 \%+ \\
\text { dexmedetomidine } 1 \mu \mathrm{\mu g} / \mathrm{kg} \text {, } \\
\text { (diluted in } 5 \mathrm{ml} \mathrm{NS} \text { ) }\end{array}$ & & & & \\
\hline \multirow[t]{2}{*}{$\begin{array}{l}\text { Sinha et al., } \\
\text { (2016) [14] }\end{array}$} & India & $18-65$ & Both & 60 & 2 & 30 & $\begin{array}{l}\text { TAP block with } 0.25 \% \\
\text { Bupivacaine }\end{array}$ & VAS & Single & $24 \mathrm{~h}$ & Diclofenac \\
\hline & & & & & & 30 & $\begin{array}{l}\text { TAP block with } 0.375 \% \\
\text { Ropivacaine }\end{array}$ & & & & \\
\hline \multirow{2}{*}{$\begin{array}{l}\text { Kamhawy } \\
\text { et al., (2017) } \\
{[15]}\end{array}$} & Egypt & $21-60$ & Both & 46 & 2 & 23 & $\begin{array}{l}\text { Unilateral subcostal TAP } \\
\text { block }\end{array}$ & VAS & Multi & $24 \mathrm{~h}$ & $\begin{array}{l}\text { PCA } \\
\text { Morphine }\end{array}$ \\
\hline & & & & & & 23 & $\begin{array}{l}\text { Unilateral thoracic } \\
\text { paravertebral block }\end{array}$ & & & & \\
\hline \multirow[t]{2}{*}{$\begin{array}{l}\text { Saxena et al., } \\
(2016)[16]\end{array}$} & India & $18-70$ & Both & 80 & 2 & 40 & $\begin{array}{l}\text { Local anesthetic infiltration } \\
\text { of surgical incision }\end{array}$ & $\begin{array}{l}\text { NRS } \\
\text { (Numerical } \\
\text { rating score) }\end{array}$ & Multi & $24 \mathrm{~h}$ & Fentanyl \\
\hline & & & & & & 40 & $\begin{array}{l}\text { Bilateral rectus sheath and } \\
\text { right TAP blocks }\end{array}$ & & & & \\
\hline \multirow{2}{*}{$\begin{array}{l}\text { Ali et al., } \\
\text { (2012) [17] }\end{array}$} & Pakistan & $35-65$ & Both & 60 & 2 & 30 & Oral pregablin $150 \mathrm{mg}$ & VAS & Single & $24 \mathrm{~h}$ & Nalbuphine \\
\hline & & & & & & 30 & Oral celecoxib 200 mg & & & & \\
\hline \multirow[t]{2}{*}{$\begin{array}{l}\text { Suseela et al., } \\
\text { (2018) [18] }\end{array}$} & India & $20-65$ & Both & 80 & 2 & 40 & $\begin{array}{l}\text { Bilateral ultrasound-guided } \\
\text { subcostal TAP block with } 20 \\
\mathrm{~mL} \text { of } 0.25 \% \text { bupivacaine }\end{array}$ & NRS & Multi & $24 \mathrm{~h}$ & $\begin{array}{l}\text { Tramadol } \\
\text { Diclofenac }\end{array}$ \\
\hline & & & & & & 40 & $\begin{array}{l}\text { Port-site infiltration with } 20 \\
\mathrm{~mL} \text { of } 0.5 \% \text { bupivacaine }\end{array}$ & & & & \\
\hline \multirow{2}{*}{$\begin{array}{l}\text { Anand et al., } \\
\text { (2017) [19] }\end{array}$} & India & $20-60$ & Both & 60 & 2 & 30 & Alprazolam & VAS & Multi & $24 \mathrm{~h}$ & Tramadol \\
\hline & & & & & & 30 & Pregabalin & & & & \\
\hline \multirow{2}{*}{$\begin{array}{l}\text { Pasha et al., } \\
\text { (2018) [20] }\end{array}$} & Nowshera & $18-65$ & Both & 90 & 2 & 45 & Gabapentin & VAS & Multi & $24 \mathrm{~h}$ & None \\
\hline & & & & & & 45 & Placebo & & & & \\
\hline \multirow[t]{2}{*}{$\begin{array}{l}\text { Khan et al., } \\
\text { (2018) [21] }\end{array}$} & pakistan & $18-60$ & Both & 126 & 2 & 63 & $\begin{array}{l}\text { Posterior TAP block with } \\
0.375 \% \text { bupivacaine }\end{array}$ & NRS & Multi & $24 \mathrm{~h}$ & Tramadol \\
\hline & & & & & & 63 & $\begin{array}{l}\text { subcostal TAP block with } \\
0.375 \% \text { bupivacaine }\end{array}$ & & & & \\
\hline \multirow[t]{2}{*}{$\begin{array}{l}\text { Jain et al., } \\
\text { (2018) [22] }\end{array}$} & India & $20-60$ & Both & 60 & 2 & 30 & $\begin{array}{l}\text { Intraperitoneal Saline with } \\
500 \mathrm{ml}\end{array}$ & NRS & single & $24 h$ & Tramadol \\
\hline & & & & & & 30 & $\begin{array}{l}\text { Intraperitoneal Bupivacaine } \\
\text { with } 20 \mathrm{ml} \text { of } 0.5 \% \text { (100 mg) }\end{array}$ & & & & \\
\hline $\begin{array}{l}\text { Amr et al., } \\
(2010)[23]\end{array}$ & Egypt & $18+$ & Both & 40 & 2 & 20 & $\begin{array}{l}\text { Preincisional thoracic } \\
\text { epidural analgesia (TEA) }\end{array}$ & VAS & Single & $24 \mathrm{~h}$ & None \\
\hline
\end{tabular}


Table 1 Characteristics of the published randomized controlled trails presented with respect to type of surgery (Continued)

\begin{tabular}{|c|c|c|c|c|c|c|c|c|c|c|c|}
\hline Authors & Country & $\begin{array}{l}\text { Age } \\
\text { Range }\end{array}$ & Gender & $\mathbf{N}$ & Groups & $\mathrm{N}$ & Modalities Studied & $\begin{array}{l}\text { Pain } \\
\text { Assessment } \\
\text { Tool }\end{array}$ & $\begin{array}{l}\text { Modalities } \\
\text { used }\end{array}$ & $\begin{array}{l}\text { Duration } \\
\text { of post- } \\
\text { operative } \\
\text { Pain } \\
\text { studied }\end{array}$ & $\begin{array}{l}\text { Rescue } \\
\text { Analgesia }\end{array}$ \\
\hline & & & & & & 20 & End of surgery TEA & & & & \\
\hline \multirow[t]{2}{*}{$\begin{array}{l}\text { Khalil et al., } \\
\text { (2017) [24] }\end{array}$} & Egypt & $20-60$ & Both & 40 & 2 & 20 & $\begin{array}{l}\text { Ultrasound-guided serratus } \\
\text { anterior plane block }\end{array}$ & VAS & Multi & $24 \mathrm{~h}$ & Morphine \\
\hline & & & & & & 20 & TEA & & & & \\
\hline \multirow[t]{2}{*}{$\begin{array}{l}\text { Dutta et al., } \\
\text { (2017) [25] }\end{array}$} & India & $18-70$ & Both & 30 & 2 & 15 & $\begin{array}{l}\text { Bolus of } 15 \mathrm{~mL} \text { of } 0.75 \% \\
\text { ropivacaine over } 3 \text { to } 5 \mathrm{~min} \text {, } \\
\text { followed by an infusion of } \\
0.2 \% \text { ropivacaine at } 0.1 \mathrm{ml} / \\
\mathrm{kg} / \text { hour }\end{array}$ & VAS & Multi & $24 \mathrm{~h}$ & Morphine \\
\hline & & & & & & 15 & $\begin{array}{l}15 \mathrm{ml} \text { of } 0.75 \% \text { ropivacaine } \\
\text { plus dexmedetomidine, } 1 \\
\mathrm{mg} / \mathrm{kg} \text { bolus over } 3 \text { to } 5 \\
\text { minutesfollowed by an } \\
\text { infusion of } 0.2 \% \text { ropivacaine } \\
\text { plus } 0.2 \mathrm{mg} / \mathrm{kg} / \text { hour of } \\
\text { dexmedetomidine at } 0.1 \mathrm{ml} / \\
\mathrm{kg} / \text { hour. }\end{array}$ & & & & \\
\hline \multirow[t]{2}{*}{$\begin{array}{l}\text { Biswas et al., } \\
\text { (2016) [26] }\end{array}$} & India & $18-60$ & Both & 60 & 2 & 30 & $\begin{array}{l}\text { Epidural: } 7.5 \mathrm{ml} \text { bolus of } \\
0.125 \% \text { Bupivacaine with } \\
50 \mu \mathrm{g} \text { Fentanyl }\end{array}$ & VAS/ FPORS & Muti & $24 \mathrm{~h}$ & Tramadol \\
\hline & & & & & & 30 & $\begin{array}{l}\text { Paravertebral } 15 \mathrm{ml} \text { bolus of } \\
0.125 \% \text { Bupivacaine with } \\
50 \mu \mathrm{g} \text { Fentanyl }\end{array}$ & & & & \\
\hline \multirow[t]{2}{*}{$\begin{array}{l}\text { Bakshi et al., } \\
\text { (2016) [27] }\end{array}$} & India & $18-75$ & Female & 71 & 2 & 36 & $\begin{array}{l}\text { Rectus sheath block with } \\
0.25 \% \text { bupivacaine }\end{array}$ & NRS & Multi & $48 \mathrm{~h}$ & Morphine \\
\hline & & & & & & 35 & $\begin{array}{l}\text { Rectus sheath block with } \\
\text { normal saline }\end{array}$ & & & & \\
\hline \multirow{2}{*}{$\begin{array}{l}\text { Dhanapal } \\
\text { et al., (2014) } \\
\text { [28] }\end{array}$} & India & $18+$ & Both & 94 & 2 & 47 & $\begin{array}{l}\text { Wound infusion with } \\
\text { Bupivacaine }\end{array}$ & VAS & Single & $48 \mathrm{~h}$ & Morphine \\
\hline & & & & & & 47 & $\begin{array}{l}\text { Wound infusion with } \mathrm{N} \\
\text { saline }\end{array}$ & & & & \\
\hline \multirow[t]{2}{*}{$\begin{array}{l}\text { Wahba et al., } \\
\text { (2014) [29] }\end{array}$} & Egypt & $59-75$ & Both & 44 & 2 & 22 & Thoracic epidural infusion & VRS & Multi & $48 \mathrm{~h}$ & $\begin{array}{l}\text { Morphine } \\
\text { (PCA) }\end{array}$ \\
\hline & & & & & & 22 & $\begin{array}{l}\text { Bilateral TAP block infusion } \\
\text { with catheter }\end{array}$ & & & & \\
\hline \multirow[t]{2}{*}{$\begin{array}{l}\text { Sethi et al., } \\
\text { (2014) [30] }\end{array}$} & India & $18-45$ & Both & 100 & 2 & 50 & $\begin{array}{l}\text { Patient controlled epidural } \\
\text { analgesia (PCEA) with } \\
\text { Ketamine + Morphine }\end{array}$ & VAS & Multiple & $48 \mathrm{~h}$ & Diclofenac \\
\hline & & & & & & 50 & PCEA Morphine & & & & \\
\hline \multirow{2}{*}{$\begin{array}{l}\text { Moawad } \\
\text { et al., (2014) } \\
{[31]}\end{array}$} & Egypt & $20-60$ & Both & 100 & 2 & 50 & $\begin{array}{l}\text { PCEA Bupivacaine + } \\
\text { Fentanyl }\end{array}$ & NRS & Multiple & $24 \mathrm{~h}$ & Fentanyl \\
\hline & & & & & & 50 & $\begin{array}{l}\text { Intravenous patient } \\
\text { controlled analgesia (PCA) } \\
\text { with Fentanyl }\end{array}$ & & & & \\
\hline \multirow[t]{2}{*}{$\begin{array}{l}\text { Patil et al., } \\
\text { (2018) [32] }\end{array}$} & India & $18-65$ & Female & 60 & 2 & 30 & $\begin{array}{l}\text { Thoracic epidural infusion } \\
\text { with } 0.125 \% \text { Ropivacaine } \\
\text { and Fentanyl } \\
0.125 \% \text { Bupivacaine and } \\
\text { Fentanyl }\end{array}$ & VAS & Multi & $24 \mathrm{~h}$ & Tramadol \\
\hline & & & & & & 30 & & & & & \\
\hline $\begin{array}{l}\text { Bharti et al., } \\
\text { (2018) [33] }\end{array}$ & India & $\begin{array}{l}\text { Adult } \\
\text { patients }\end{array}$ & Both & 40 & 2 & 20 & $\begin{array}{l}50 \mu \mathrm{g} \text { Dexmedetomidine } \\
\text { with } 10 \mathrm{ml} \text { of } 0.125 \% \\
\text { Bupivacaine in thoracic } \\
\text { epidural }\end{array}$ & VAS & Multi & $24 \mathrm{~h}$ & Diclofenac \\
\hline
\end{tabular}


Table 1 Characteristics of the published randomized controlled trails presented with respect to type of surgery (Continued)

\begin{tabular}{|c|c|c|c|c|c|c|c|c|c|c|c|}
\hline Authors & Country & $\begin{array}{l}\text { Age } \\
\text { Range }\end{array}$ & Gender & $\mathbf{N}$ & Groups & $\mathbf{N}$ & Modalities Studied & $\begin{array}{l}\text { Pain } \\
\text { Assessment } \\
\text { Tool }\end{array}$ & $\begin{array}{l}\text { Modalities } \\
\text { used }\end{array}$ & $\begin{array}{l}\text { Duration } \\
\text { of post- } \\
\text { operative } \\
\text { Pain } \\
\text { studied }\end{array}$ & $\begin{array}{l}\text { Rescue } \\
\text { Analgesia }\end{array}$ \\
\hline & & & & & & 20 & $\begin{array}{l}50 \mu \mathrm{g} \text { Fentanyl with } 10 \mathrm{ml} \\
0.125 \% \text { Bupivacaine in } \\
\text { thoracic epidural }\end{array}$ & & & & \\
\hline \multirow{2}{*}{$\begin{array}{l}\text { Alvi et al., } \\
\text { (2017) [34] }\end{array}$} & Pakistan & $18-50$ & Both & 200 & 2 & 100 & TAP block & NRS & Single & $24 \mathrm{~h}$ & None \\
\hline & & & & & & 100 & Placebo block & & & & \\
\hline \multirow[t]{2}{*}{$\begin{array}{l}\text { Patel et al., } \\
\text { (2018) [35] }\end{array}$} & India & $\begin{array}{l}20 \text { to } \\
65\end{array}$ & Both & 60 & 2 & 30 & $\begin{array}{l}\text { TAP block with Ropivacaine } \\
(0.5 \%) 20 \mathrm{ml}\end{array}$ & VAS & Multi & $24 \mathrm{~h}$ & Diclofenac \\
\hline & & & & & & 30 & Spinal anaesthesia & & & & \\
\hline \multirow[t]{2}{*}{$\begin{array}{l}\text { Mishra et al., } \\
\text { (2018) [36] }\end{array}$} & India & $18-60$ & Both & 60 & 2 & 30 & $\begin{array}{l}\text { Thoracic paravertebral block } \\
\text { ( } 20 \mathrm{~mL} 0.25 \% \text { bupivacaine) }\end{array}$ & VAS & Multi & $24 \mathrm{~h}$ & Tramadol \\
\hline & & & & & & 30 & IV PCA with fentanyl & & & & \\
\hline \multirow[t]{2}{*}{$\begin{array}{l}\text { Bharti et al., } \\
\text { (2011) [37] }\end{array}$} & India & $18-60$ & Both & 40 & 2 & 20 & $\begin{array}{l}\text { TAP Block with } 20 \mathrm{~mL} \text { of } \\
0.25 \% \text { bupivacaine }\end{array}$ & VAS & Single & $24 \mathrm{~h}$ & Morphine \\
\hline & & & & & & 20 & TAP Block with Saline & & & & \\
\hline \multirow{2}{*}{$\begin{array}{l}\text { Chotton et al., } \\
\text { (2014) [38] }\end{array}$} & India & $18-60$ & Female & 90 & 2 & 45 & Pregabalin 150 mg & VAS & Multi & $24 \mathrm{~h}$ & Ketorolac \\
\hline & & & & & & 45 & Placebo & & & & \\
\hline $\begin{array}{l}\text { Badawy et al., } \\
\text { (2015) [39] }\end{array}$ & Egypt & $40-70$ & Female & 60 & 3 & $\begin{array}{l}20 \\
20 \\
20\end{array}$ & $\begin{array}{l}\text { Oral gabapentin } 800 \text { mg } \\
\text { Gabapentin } 800 \mathrm{mg}+ \\
\text { Dexamethasone } 8 \mathrm{mg} \\
\text { Placebo }\end{array}$ & VAS & Multi & $24 \mathrm{~h}$ & Meperidine \\
\hline $\begin{array}{l}\text { Bashandy } \\
\text { et al., (2015) } \\
\text { [40] }\end{array}$ & Egypt & Adult & Female & 120 & 2 & $\begin{array}{l}60 \\
60\end{array}$ & $\begin{array}{l}\text { Ultrasound-guided Pecs } \\
\text { block } \\
\text { Control }\end{array}$ & VAS & Single & $24 \mathrm{~h}$ & Morphine \\
\hline $\begin{array}{l}\text { Hetta et al., } \\
\text { (2016) [41] }\end{array}$ & Egypt & Adult & Female & 111 & 4 & $\begin{array}{l}28 \\
27 \\
26 \\
30\end{array}$ & $\begin{array}{l}\text { Pregabalin } 75 \mathrm{mg} \\
\text { Pregabalin } 150 \mathrm{mg} \\
\text { Pregabalin } 300 \mathrm{mg} \\
\text { Placebo Capsule }\end{array}$ & VAS & Single & $24 \mathrm{~h}$ & Morphine \\
\hline $\begin{array}{l}\text { Kasimahanti } \\
\text { et al.,(2016) } \\
{[42]}\end{array}$ & India & $18-60$ & Female & 58 & 2 & $\begin{array}{l}28 \\
30\end{array}$ & $\begin{array}{l}\text { Single-level, unilateral } \\
\text { ultrasound-guided TPVB at } \\
\text { T4 level using } 0.3 \mathrm{~mL} / \mathrm{kg} \text { of } \\
0.5 \% \text { ropivacaine } \\
\text { Double-level, unilateral } \\
\text { ultrasound-guided TPVB at } \\
\text { T2 and T5 level using a total } \\
\text { volume of } 0.3 \mathrm{~mL} / \mathrm{kg} \text { of } 0.5 \% \\
\text { ropivacaine }\end{array}$ & NRS & Single & $24 \mathrm{~h}$ & Diclofenac \\
\hline \multirow[t]{2}{*}{$\begin{array}{l}\text { Kulhari et al., } \\
\text { (2016) [43] }\end{array}$} & India & $18-65$ & Female & 40 & 2 & 20 & $\begin{array}{l}\text { TPVB with ropivacaine } 0.5 \% \text {, } \\
25 \mathrm{ml} \text {, }\end{array}$ & VAS & Single & $24 \mathrm{~h}$ & Morphine \\
\hline & & & & & & 20 & $\begin{array}{l}\text { PECS II block with } \\
\text { ropivacaine } 0.5 \%\end{array}$ & & & & \\
\hline \multirow[t]{2}{*}{$\begin{array}{l}\text { Gupta et al., } \\
\text { (2017) [44] }\end{array}$} & India & $18-65$ & Female & 50 & 2 & 25 & Paravertebral block & VAS & Multi & $72 \mathrm{~h}$ & $\begin{array}{l}\text { PCA } \\
\text { Morphine }\end{array}$ \\
\hline & & & & & & 25 & Serratus Plane block & & & & \\
\hline \multirow[t]{3}{*}{$\begin{array}{l}\text { Bhuvaneswari } \\
\text { et al.,(2012) } \\
\text { [45] }\end{array}$} & India & Adult & Female & 48 & 4 & 12 & $\begin{array}{l}\text { Paravertebral block with } \\
0.25 \% \text { bupivacaine with } \\
\text { epinephrine } 5 \mathrm{mcg} / \mathrm{ml}\end{array}$ & VAS & Single & $24 \mathrm{~h}$ & Morphine \\
\hline & & & & & & 12 & $\begin{array}{l}\text { Paravertebral block with } \\
0.25 \% \text { bupivacaine }+ \\
\text { epinephrine } 5 \mathrm{mcg} / \mathrm{ml} \text { with } \\
2 \mathrm{mcg} / \mathrm{ml} \text { fentanyl }\end{array}$ & & & & \\
\hline & & & & & & 12 & $\begin{array}{l}\text { Paravertebral block with } \\
0.5 \% \text { bupivacaine }+ \\
\text { epinephrine } 5 \mathrm{mcg} / \mathrm{ml} \text { or }\end{array}$ & & & & \\
\hline
\end{tabular}


Table 1 Characteristics of the published randomized controlled trails presented with respect to type of surgery (Continued)

\begin{tabular}{|c|c|c|c|c|c|c|c|c|c|c|c|}
\hline Authors & Country & $\begin{array}{l}\text { Age } \\
\text { Range }\end{array}$ & Gender & $\mathbf{N}$ & Groups & $\mathbf{N}$ & Modalities Studied & $\begin{array}{l}\text { Pain } \\
\text { Assessment } \\
\text { Tool }\end{array}$ & $\begin{array}{l}\text { Modalities } \\
\text { used }\end{array}$ & $\begin{array}{l}\text { Duration } \\
\text { of post- } \\
\text { operative } \\
\text { Pain } \\
\text { studied }\end{array}$ & $\begin{array}{l}\text { Rescue } \\
\text { Analgesia }\end{array}$ \\
\hline & & & & & & & isotonic saline & & & & \\
\hline & & & & & & 12 & Noraml Salin & & & & \\
\hline \multirow{3}{*}{$\begin{array}{l}\text { Mahran et al., } \\
\text { (2015) [46] }\end{array}$} & Egypt & $18-65$ & Female & 90 & 3 & 30 & Pregabalin 150 mg oral & VAS & Multi & $24 \mathrm{~h}$ & Morphine \\
\hline & & & & & & 30 & $\begin{array}{l}\text { Placebo capsule (oral) and } \\
0.5 \mathrm{mg} / \mathrm{kg} \text { ketamine IV }\end{array}$ & & & & \\
\hline & & & & & & 30 & Placebo & & & & \\
\hline \multirow[t]{2}{*}{$\begin{array}{l}\text { Kundra et al., } \\
\text { (2013) [47] }\end{array}$} & India & Adult & Female & 120 & 2 & 60 & Paravertebral block & VAS & Multi & $24 \mathrm{~h}$ & $\begin{array}{l}\text { No rescue } \\
\text { analgesia }\end{array}$ \\
\hline & & & & & & 60 & Interpleural block & & & & \\
\hline \multirow{2}{*}{$\begin{array}{l}\text { M. Neetu } \\
\text { et al., (2018) } \\
\text { [48] }\end{array}$} & India & $18-70$ & Female & 60 & 2 & 30 & PECS block & VAS & Multi & $24 \mathrm{~h}$ & None \\
\hline & & & & & & 30 & Placebo & & & & \\
\hline \multirow{2}{*}{$\begin{array}{l}\text { Mukherjee } \\
\text { et al., (2018) } \\
\text { [49] }\end{array}$} & India & $35-60$ & female & 88 & 2 & 44 & Ropivacaine (0.5\%) & VAS & single & $48 \mathrm{~h}$ & Diclofenac \\
\hline & & & & & & 44 & Dexmedetomidine $1 \mu / \mathrm{kg}$ & & & & \\
\hline \multirow[t]{2}{*}{$\begin{array}{l}\text { Megha et al., } \\
\text { (2018) [50] }\end{array}$} & India & $18-60$ & Female & 47 & 2 & 23 & $\begin{array}{l}\text { Paravertebral block with } 20 \\
\text { ml bupivacaine } 0.25 \% \text { with } \\
\text { morphine } 3 \mathrm{mg}\end{array}$ & NRS & Multi & $24 \mathrm{~h}$ & Diclofenac \\
\hline & & & & & & 24 & Dexmedetomidine 1 mg/kg & & & & \\
\hline \multirow{2}{*}{$\begin{array}{l}\text { Manzoor } \\
\text { et al., (2018) } \\
{[51]}\end{array}$} & India & $18-70$ & Female & 60 & 2 & 30 & $\begin{array}{l}\text { PECS I block with } 30 \mathrm{ml} \\
0.25 \% \text { bupivacaine }\end{array}$ & VAS & single & $24 \mathrm{~h}$ & Diclofenac \\
\hline & & & & & & 30 & $\begin{array}{l}\text { PECS I block with } 10 \mathrm{ml} \\
0.25 \% \text { bupivacaine with } \\
\text { dexmedetomidine }\end{array}$ & & & & \\
\hline \multirow[t]{2}{*}{$\begin{array}{l}\text { Kumar et al., } \\
\text { (2018) [52] }\end{array}$} & India & $?$ & Female & 50 & 2 & 25 & $\begin{array}{l}\text { Opioids and non-steroidal } \\
\text { anti-inflammatory drug }\end{array}$ & VAS & Multi & $24 \mathrm{~h}$ & Tramadol \\
\hline & & & & & & 25 & $\begin{array}{l}\text { PECS I with } 0.25 \% \\
\text { bupivacaine }\end{array}$ & & & & \\
\hline \multirow[t]{2}{*}{$\begin{array}{l}\text { Bharti et al., } \\
\text { (2015) [53] }\end{array}$} & India & $20-60$ & Both & 54 & 2 & 27 & $\begin{array}{l}\text { Supraclavicular block with } \\
1 \mathrm{\mu g} / \mathrm{kg} \text { of } \\
\text { Dexmedetomidine along } \\
\text { with equal volumes of } 0.75 \% \\
\text { ropivacaine and } 2 \% \\
\text { lidocaine with adrenaline. }\end{array}$ & VAS & Single & $24 \mathrm{~h}$ & $\begin{array}{l}\text { Tramadol } \\
\text { Diclofenac }\end{array}$ \\
\hline & & & & & & 27 & $\begin{array}{l}0.75 \% \text { Ropivacaine and } 2 \% \\
\text { Lidocaine with adrenaline ( } 1 \text { : } \\
2,00,000)\end{array}$ & & & & \\
\hline \multirow[t]{2}{*}{$\begin{array}{l}\text { Kumar et al., } \\
\text { (2014) [54] }\end{array}$} & India & $18-60$ & Both & 30 & 2 & 15 & $\begin{array}{l}\text { Ultrasound-guided stellate } \\
\text { ganglion block with } 2 \% \\
\text { Lidocaine }\end{array}$ & VAS & Single & $24 \mathrm{~h}$ & $\begin{array}{l}\text { PCIA Used } \\
\text { [No } \\
\text { Rescue] }\end{array}$ \\
\hline & & & & & & 15 & $\begin{array}{l}\text { Ultrasound-guided stellate } \\
\text { ganglion block with } 0.9 \% \\
\text { Saline }\end{array}$ & & & & \\
\hline \multirow[t]{2}{*}{$\begin{array}{l}\text { Mullaji et al., } \\
\text { (2010) [55] }\end{array}$} & India & $50-80$ & Both & 40 & 2 & 20 & $\begin{array}{l}\text { Combined spinal epidural + } \\
\text { local anesthetic infiltration }\end{array}$ & VAS & Single & $24 \mathrm{~h}$ & None \\
\hline & & & & & & 20 & $\begin{array}{l}\text { Combined spinal epidural } \\
\text { with no local anesthetic } \\
\text { infiltration }\end{array}$ & & & & \\
\hline \multirow[t]{2}{*}{$\begin{array}{l}\text { Khanna et al., } \\
\text { (2017) [56] }\end{array}$} & India & $40-60$ & Both & 90 & 3 & 30 & $\begin{array}{l}\text { Epidural with ropivacaine } \\
0.1 \%\end{array}$ & VAS & Multi & $36 \mathrm{~h}$ & PCEA \\
\hline & & & & & & 30 & $\begin{array}{l}\text { Epidural with ropivacaine } \\
0.1 \% \text { with fentanyl } 2.5 \mu \mathrm{g} / \mathrm{mL}\end{array}$ & & & & \\
\hline
\end{tabular}


Table 1 Characteristics of the published randomized controlled trails presented with respect to type of surgery (Continued)

\begin{tabular}{|c|c|c|c|c|c|c|c|c|c|c|c|}
\hline Authors & Country & $\begin{array}{l}\text { Age } \\
\text { Range }\end{array}$ & Gender & $\mathbf{N}$ & Groups & $\mathrm{N}$ & Modalities Studied & $\begin{array}{l}\text { Pain } \\
\text { Assessment } \\
\text { Tool }\end{array}$ & $\begin{array}{l}\text { Modalities } \\
\text { used }\end{array}$ & $\begin{array}{l}\text { Duration } \\
\text { of post- } \\
\text { operative } \\
\text { Pain } \\
\text { studied }\end{array}$ & $\begin{array}{l}\text { Rescue } \\
\text { Analgesia }\end{array}$ \\
\hline & & & & & & 30 & $\begin{array}{l}\text { Epidural with ropivacaine } \\
0.0625 \% \text { with fentanyl } \\
2.5 \mu \mathrm{g} / \mathrm{mL}\end{array}$ & & & & \\
\hline \multirow[t]{3}{*}{$\begin{array}{l}\text { Anis et al., } \\
\text { (2011) [57] }\end{array}$} & Egypt & $18-60$ & Both & 60 & 3 & 20 & $\begin{array}{l}\text { Lumbar plexus block with } \\
15 \mathrm{ml} \text { bupivacaine } 0.25 \%+ \\
\text { clonidine }\end{array}$ & VAS & Single & $24 \mathrm{~h}$ & Morphine \\
\hline & & & & & & 20 & $\begin{array}{l}\text { Lumbar plexus block with } \\
\text { bupivacaine } 0.25 \%+ \\
\text { clonidine }\end{array}$ & & & & \\
\hline & & & & & & 20 & No Block & & & & \\
\hline \multirow{4}{*}{$\begin{array}{l}\text { Sawhney } \\
\text { et al., (2015) } \\
\text { [58] }\end{array}$} & India & Adult & Both & 100 & 4 & 25 & $\begin{array}{l}\text { Epidural with } 0.2 \% \\
\text { Ropivacaine }\end{array}$ & VAS & Single & $24 \mathrm{~h}$ & Tramadol \\
\hline & & & & & & 25 & $\begin{array}{l}\text { Epidural with } 0.1 \% \\
\text { Ropivacaine+Fentanyl } 2 \text { mg/ } \\
\text { ml }\end{array}$ & & & & \\
\hline & & & & & & 25 & $\begin{array}{l}\text { Epidural with } 0.2 \% \\
\text { Bupivacaine }\end{array}$ & & & & \\
\hline & & & & & & 25 & $\begin{array}{l}\text { Epidural with Bupivacaine } \\
0.1 \% \text { with Fentanyl } 2 \mu \mathrm{g} / \mathrm{ml} \text {. }\end{array}$ & & & & \\
\hline \multirow[t]{2}{*}{$\begin{array}{l}\text { Trabelsi et al., } \\
\text { (2017) [59] }\end{array}$} & Tunisia & $>18$ & Both & 60 & 2 & 30 & $\begin{array}{l}\text { Suprascapular block + } \\
\text { supraclavicular block }\end{array}$ & VAS & Multi & $24 \mathrm{~h}$ & Morphine \\
\hline & & & & & & 30 & Interscalene block & & & & \\
\hline \multirow[t]{2}{*}{$\begin{array}{l}\text { Meghana } \\
\text { et al., (2017) } \\
\text { [60] }\end{array}$} & India & $20-65$ & Both & 70 & 2 & 35 & $\begin{array}{l}0.125 \% \text { bupivacaine and } 2 \\
\mathrm{mg} / \mathrm{ml} \mathrm{fentanyl} \mathrm{epidural} \\
\text { infusion }\end{array}$ & NRS & Single & $48 \mathrm{~h}$ & Tramadol \\
\hline & & & & & & 35 & $\begin{array}{l}0.2 \% \text { ropivacaine and } 2 \mathrm{mg} / \\
\mathrm{ml} \text { fentanyl as epidural } \\
\text { infusion }\end{array}$ & & & & \\
\hline \multirow[t]{3}{*}{$\begin{array}{l}\text { Thakur et al., } \\
\text { (2015) [61] }\end{array}$} & India & $18-60$ & Both & 67 & 3 & 22 & $\begin{array}{l}\text { Axillary brachial plexus block } \\
\text { with bupivacine, lignocaine, } \\
\text { adrenaline and } \\
\text { buprenorphine + IM placebo }\end{array}$ & VAS & Single & $24 \mathrm{~h}$ & Diclofenac \\
\hline & & & & & & 23 & $\begin{array}{l}\text { Axillary brachial plexus block } \\
\text { with bupivacine, lignocaine, } \\
\text { adrenaline and placebo + IM } \\
\text { buprenorphine }\end{array}$ & & & & \\
\hline & & & & & & 22 & $\begin{array}{l}\text { Axillary brachial plexus block } \\
\text { with bupivacine, lignocaine, } \\
\text { adrenaline and placebo + IM } \\
\text { placebo }\end{array}$ & & & & \\
\hline \multirow{2}{*}{$\begin{array}{l}\text { Mostafa et al., } \\
\text { (2018) [62] }\end{array}$} & Egypt & $50-70$ & Both & 60 & 2 & 30 & Levobupivacaine $0.125 \%$ & VAS & Multi & $24 \mathrm{~h}$ & None \\
\hline & & & & & & 30 & IV fentanyl $20 \mu \mathrm{g} / \mathrm{ml}$ & & & & \\
\hline
\end{tabular}

RCTs [29-33]. In one trial it was compared with continuous infusion through bilateral TAP block [68]. Another trial had compared patient controlled epidural analgesia (PCEA) with a combination of morphine and ketamine with morphine alone [30]. One trial compared bupivacaine-fentanyl PCEA with fentanyl patient controlled intravenous analgesia (PCIA) [31]. Another compared bupivacaine and fentanyl with ropivacaine and fentanyl infusion through thoracic epidural [32]. Yet another trial employing thoracic epidural compared dexmedetomidine and fentanyl as adjuncts to local anaesthetic [33]. TAP block was compared with placebo (normal saline) in three RCTs [34, 35, 37]. Thoracic paravertebral block was compared with IV PCA in another trial [45]. Multimodal analgesia was employed in all trials along with the modalities being studied. Morphine, pethidine, or diclofenac was used for rescue analgesia in these RCTs. 


\section{Hysterectomy}

In 2 RCTs $[38,39]$ performed on patients undergoing TAH, 150 patients were included, aged 18-70 years (Table 1). Preoperative pregabalin was compared with placebo in one of the trials, while gabapentin with or without dexamethasone was compared with placebo in the other. The effect of these drugs on postoperative analgesic consumption was studied. Rescue analgesia was provided with ketorolac [38] or pethidine [39].

\section{Breast Cancer surgery}

A total of 13 RCTs [40-52] were included. In 11 trials, multimodal analgesia was used with some form of regional analgesia (Table 1). In one trial [41], oral pregabalin was used pre-operatively for reducing postoperative pain and morphine consumption in patients undergoing mastectomy. In another trail [46], preoperative oral pregabalin $(150 \mathrm{mg})$ was compared with intravenous ketamine (0.5 mg.kg-1) at induction of anaesthesia and showed reduction in postoperative opioid consumption without increasing sedation and with a good safety profile. In seven RCTs, thoracic paravertebral block (TPVB) was used for intra and post-operative pain management. In one trial [42], single level TPVB was compared with block at two different levels. Bupivacaine was used with epinephrine in one trial [45], while one used ropivacaine with dexmedetomidine [49] as adjuvants with better post-operative pain relief. One trial compared morphine and dexmedetomidine with bupivacaine in TPVB [50]. In one trial, TPVB was compared with intrapleural block [47]. In two trials, TPVB was compared with newer blocks like PECS II block [43] and serratus plane block [44]. In two RCTs [40, 48] ultrasound-guided PECS I \& II blocks were used for pain management. In one trial [42], bupivacaine $0.25 \%$ was used with or without dexmedetomidine for PECS I \& II blocks. In one study [52], ultrasound guided PECS I \& II blocks were compared with intravenous opioids and NSAIDS.

\section{Orthopedic procedures}

A total of 10 RCTs [53-62] fulfilled the inclusion criteria. Four RCTs included upper limb procedures (Table 1). In these trials, peripheral nerve blocks (supraclavicular, stellate ganglion, supraclavicular and axillary nerve blocks) were compared either with different concentrations of local anaesthetic agents or with other modalities such as intramuscular narcotics [53, 54, 59, 61]. In lower limb procedures, 2 RCTs were performed on total knee arthroplasty and the modalities used were sub-arachnoid block, lumbar epidural and intra articular infiltration of local anaesthetic drugs. Different local anaesthetic agents were compared in different concentrations $[55,56]$ Two RCTs were done on hip surgery $[57,62]$ and two were on generalized lower limb procedures [58,60]. Lumbar plexus block, lumbar epidural, fascia iliaca compartment block and intravenous narcotics were compared.

\section{Discussion}

Ineffective pain management in the postoperative period leads to untoward consequences like slower recovery and increased cost of care. Optimal pain management modalities enable earlier mobilization and ease of performing physical therapy with resultant early functional recovery. Recent decades have seen a surge of research directed towards improvement in the quality of postoperative pain relief with special focus on procedure specific pain management. Bulk of this research has originated from the developed world.

Systematic reviews are now being carried out in health care systems to get the best evidence for decision making and to subsequently include the researched modality/intervention in the clinical practice. Two main purposes of a systematic review are to establish the extent to which existing research has progressed toward explaining a problem, and to clarify the extent to which this evidence explains a new or existing question. The purpose of this systematic review is to deliver a meticulous summary of all the available RCTs performed in LMIC over the last decade for the management of postoperative pain in adult patients, to scrutinize the types of modalities being used in LMIC for postoperative pain relief, and to compare these modalities with those being used in the developed world.

The PROSPECT is an international collaboration of anaesthesiologists and surgeons. The PROSPECT aims to provide healthcare professionals with practical procedure-specific pain management recommendations formulated in a way that facilitates clinical decisionmaking across all the stages of the perioperative period [63]. For postoperative pain management for laparoscopic cholecystectomy procedure, PROSPECT [64] recommends multimodal analgesia including wound infiltration with long acting local anaesthetic (LA), intraperitoneal infiltration of LA or both, paracetamol, COX2 selective inhibitors, NSAIDs, and opioids for rescue analgesia. Four out of 11 RCTs from LMIC used regional blocks, which are neither recommended, nor notrecommended in PROSPECT. However, that can be due to PROSPECT recommendations being formulated in 2005 while use of abdominal wall blocks is rather a recent phenomenon. Intraperitoneal infiltration of LA was studied in three instances. One trial compared LA infiltration of incisional wounds with abdominal plane blocks. Oral Pregabalin, Cox-2 inhibitor, and Gabapentin were also studied. Majority [five] RCTs used TAP blocks for the study group, intraperitoneal infiltration with LA in three, and gabapentinoids in three. Usual care or control groups received either TAP block at a different level 
than the study group [subcostal vs. conventional], different drugs or different concentrations of the same drugs. LA infiltration of the surgical wounds was employed in two control groups while celecoxib and alprazolam were used for two. One trial used placebo for control group in place of the studied modality. All trials used multimodal analgesia for pain management overall, which is according to the international recommendations.

Thoracotomy is considered one of the most painful surgical procedures. Inadequate pain relief after thoracotomy can result in postoperative pulmonary complications. Considering multifactorial nature of thoracotomy, a single modality cannot provide adequate pain control. The management of pain after thoracotomy requires a multimodal approach incorporating regional and systemic analgesia to targets multiple sites [65]. Regional analgesia is highly recommended with non-steroidal anti-inflammatory drugs (NSAIDs), paracetamol, opioids and other adjuvants for the pain following thoracotomy. Analgesic effect of paracetamol with NSAIDs is additive. None of the RCTs used NSAIDs and paracetomol for post thoracotomy pain.

Continuous thoracic epidural analgesia is recommended by PROSPECT for postoperative pain management following laparotomy, ensuring an appropriate level according to the site of incision [63]. A combination of local anaesthetic agent and an opioid for the epidural infusion has better analgesic efficacy compared to either agent alone. When the patient does not receive an epidural due to contraindication or lack of feasibility, strong opioids using intravenous patient controlled analgesia (IV PCA) are recommended for high intensity pain as part of a multimodal regime. Multimodal analgesia in such cases may include non-steroidal anti-inflammatory drugs, paracetamol and intravenous lignocaine. Preperitoneal infusion of local anesthetic is recommended in patients who have not received an epidural. The RCTs $\mathrm{s}$ performed on postoperative pain relief in LMIC have employed multimodal analgesia in all cases as recommended for these procedures. Thoracic epidural was used in five of the 11 trials, while PCA was used in two. Majority RCTs [five] used thoracic epidural followed by TAP block [three]. In the case of usual care or control groups, thoracic epidural was employed but using either a different drug or a different concentration, while IV PCA was used for two control groups. Placebo was used in the control groups in three studies, replacing the studied modality. Abdominal wall blocks were employed in five studies, which are not part of the PROSPECT recommendations. As pointed out above, abdominal wall blocks have come in vogue after the PROSPECT recommendations. Since multiple and varied pain relieving modalities have been employed and compared in different studies, it is difficult to compare the results with the current recommendations.

Multimodal analgesia is recommended for postoperative pain relief following total abdominal hysterectomy (TAH). For severe pain, PROSPECT recommends strong opioids using PCA along with NSAIDs such as Diclofenac. Opioids can be administered as a continuous infusion, when PCA is not feasible. Weak opioids can be substituted along with paracetamol and NSAIDs when pain decreases to moderate intensity [66]. Though epidural analgesia is not recommended for routine use, it is considered useful for high-risk patients undergoing TAH. In both the RCTs conducted in LMIC on posthysterectomy pain, multimodal analgesia was not employed, rather pre-emptive analgesic effect of gabapentin (plus dexamethasone) or pregabalin was studied on consumption of a single postoperative analgesic agent (ketorolac [38] or pethidine [39]. Both used placebo for usual care groups.

A multimodal approach has been recommended for perioperative pain management in major breast cancer surgery. A successful multimodal approach requires coordination between surgical, anaesthesia, and nursing staff throughout perioperative period. Recent recommendations [67] are to use antiepileptic medication or gabapentinoids (gabapentin or pregabalin), paracetamol, and regional nerve blocks (paravertebral blocks, PEC blocks, or thoracic epidural injection), wound infiltration with LA at the end, NSAIDs, and intermittent shortacting opioids. This regimen should be continued for up to 1 week after surgery. Other classes of medications can also be used such as, intravenous lignocaine, $\mathrm{N}$-methylD-aspartate (NMDA) antagonists such as ketamine and magnesium, alpha-2-adrenergic antagonists clonidine and dexmedetomidine. Glucocorticoids such as dexamethasone have been used to minimize postoperative pain, nausea and vomiting.

PROSPECT recommendations for non-cosmetic major breast surgery [68] include paravertebral block, gabapentinoids, COX-2-selective inhibitors, paracetamol, IV dexamethasone, intercostal nerve block plus other regional techniques (TPVB), NSAIDs, strong opioids, (for high intensity pain) or weak opioids for moderate to low intensity pain, paracetamol alone or in combination with other nonopioid analgesics for low to moderate intensity pain. Majority of the RCTs [seven] employed thoracic paravertebral blocks, followed by PECS I and II block [four]. Though regional techniques were employed, there was a gap in comparison to the recent recommendations, such as preoperative use of antiepileptic medication or gabapentinoids, paracetamol and, intraoperative wound infiltration with LA, NSAIDs, and intermittent short-acting opioids. Usual care or control groups used different drugs or 
different concentrations of the drug for TPVB and PECS I and II blocks. In LMIC, incidence of breast cancer is rising and increasing number of patients are undergoing these procedures. Healthcare teams hence are required to develop and follow multimodal pain management protocols as per recent recommendations to provide quality care to their patients. Multimodal preventive analgesia regimen needs to be followed in patients scheduled for major breast cancer surgery.

Moderate to severe pain is not uncommon after orthopedic procedures, especially after joint replacement surgeries. If not adequately controlled, there is a high probability of developing persistent post-surgical pain. Two commonly performed procedures in the lower limb are total knee arthroplasty (TKA) and hip replacement surgery. In RCTs carried out in LMIC, the modalities used for TKA were local anaesthetic infiltration in joint space, lumbar epidural, combined spinal epidural, and lumbar plexus block. According to the PROSPECT recommendations [68] for TKA, peripheral neural block is strongly recommended for best post-operative pain management. Epidural block is only recommended for patients having increased risk of cardio-pulmonary complications and in those cases where general anaesthesia is contraindicated due to increased risk of morbidity; otherwise epidural is not recommended for postoperative analgesia after TKA. Intra-articular infiltration of local anaesthetics is also not recommended because of inconsistent evidence. Similarly ASA (American Society of Anesthesiologists) strongly recommends the use of peripheral nerve blocks, either continuous or single shot, after TKA and hip surgeries [69]. Hence in LMIC, the post-operative pain management practices for lower limb surgeries are not according to the evidence based recommended methods, which is probably due to lack of expertise in performing peripheral nerve blocks, lack of knowledge, or due to a large patient volume.

On the contrary, for upper limb and shoulder surgeries the studies done in LMIC have shown that peripheral nerve blocks were used for post-operative pain management. ASA also strongly recommends peripheral nerve blocks for upper extremities and shoulder surgery. However there is no recommendation by PROSPECT for upper limb surgeries as yet. Hence the pain management strategies for upper limb surgeries in LMIC seem to be consistent with the current practice of the developed countries.

This systematic review addresses the post-operative pain management in LMIC for the first time. A potential limitation of this review is the inclusion of last 10 years studies with a wide range of clinical outcomes. The included studies were conducted in only three LMICs out of total 47 listed. It is difficult to estimate the direct cost of postoperative pain in LMICs in included studies due to non- availability of the data regarding resumption of routine functions. Though the review shows a congruence of RCTs being carried out in the LMICs with internationally available recommendations and guidelines in majority of the instances, it is pertinent to realize that clinical practices on the ground may not reflect this. The findings of this review should be interpreted cautiously as majority of RCTs are small. This indeed is another limitation of the review. Placebo was used in four RCTs for the control groups, replacing study drug/intervention. Although there were other analgesia options in the multimodal regimen being used to treat pain, use of placebo is outdated and not encouraged for pain research. This systemic review, based on RCTs on postoperative pain management in LMICs, identified numerous research gaps in the included small sample sized low-quality studies. Authors believe that there is an urgent need to conduct research on practice gaps regarding the use of cost-effective evidencebased management of postoperative pain in LMICs.

\section{Conclusion}

Three billion people live in LMICs out of a total world population of 7.53 billion. Scientific literature is very scant coming from the part of the world housing nearly half its population. Guidelines and recommendations are formulated based on research carried out entirely in the other half, yet LMICs try to follow them. The current review shows the same trend. Multimodal analgesia is being used for majority of the procedures; while use of regional analgesia as part of multi-modal analgesia was common however this research snapshot (of only three countries) may not exactly reflect the clinical practices in all 47 countries.

Systematic reviews do not merely determine what is being done but also identify and document knowledge gaps in the literature. These gaps then can be used to shape future research agendas in the LMICs related to any question, for example post-operative pain. It is essential to realize that improved health care practices require evidence based research carried out in LMIC to guide development of relevant and contextual standards of care. The authors strongly recommend the conduct of more RTCs in LMIC based on the available resources for postoperative pain management rather than conducting them in accordance with international guidelines of developed countries.

\section{Abbreviations}

LMIC: low and middle-income countries; GNI: gross national income; RCTs: randomized control trials; CASP: critical appraisal skill program; PCEA: patient controlled epidural analgesia; PCIA: patient controlled intravenous analgesia; TAH: total abdominal hysterectomy; APS: acute pain services; TKA: total knee arthroplasty; ASA: American Society of

Anesthesiologists; TAP: transversus abdominis plane; SAPB: serratus anterior plane block; TPVB: thoracic paravertebral block; LA: local anaesthetic; NSAI D: non -steroidal anti-inflammatory drugs; NMDA: N-methyl-D-aspartate 


\section{Acknowledgments}

The authors wish to thank Ms. Seharish Sher Ali and Ms. Nausheen Abbas for providing administrative support during the development of the manuscript and its electronic submission.

\section{Authors' contributions}

All authors have read and approved the manuscript. GA: Conceptualization the idea, assigned the tasks to all co-authors \& manuscript writing and review. AA, RIK: Critical appraisal of selected articles \& manuscript writing and review. ASS, AR \& RK: Contributed in literature search, critical and appraisal of selected articles. SAR, KM: Literature search, Biostatistics support, Organization of data and formatted table of systemic review.

\section{Funding}

Nil

\section{Availability of data and materials}

All data generated or analysed during this study are included in this published article.

\section{Declarations}

Ethics approval and consent to participate Not Applicable.

\section{Consent for publication}

Not applicable.

\section{Competing interests}

The authors declared no potential competing interest with respect to the research, authorship and/or publication of this article.

\section{Author details}

'Department of Anaesthesiology, 2nd floor Private Wing, Aga Khan University, P.O. Box 3500, Stadium Road, Karachi 74800, Pakistan. ${ }^{2}$ Faculty of Health Sciences, Aga Khan University, P.O. Box 3500, Stadium Road, Karachi, Pakistan

Received: 5 August 2020 Accepted: 11 May 2021 Published online: 25 May 2021

\section{References}

1. Junkins S, Goloshchapov D, Sindt JE. Pain in low resource environments. Curr Anesthesiol Rep. 2017;7(1):23-9. https://doi.org/10.1007/s40140-017-01 93-x.

2. Brennan F, Carr DB, Cousins M. Pain management: a fundamental human right. Anesth Analg. 2007;105(1):205-21. https://doi.org/10.1213/01.ane. 0000268145.52345 .55 .

3. Lipman AG. Pain as a human right: the 2004 global day against pain. J Pain Palliat Care Pharmacother. 2005;19(3):85-100.

4. King NB, Fraser V. Untreated pain, narcotics regulation, global health ideologies. PLoS Med. 2013;10(4):e1001411. www.plosmedicine.org. https:// doi.org/10.1371/journal.pmed.1001411.

5. International Narcotics Control Board. Narcotics Drugs. Technical reports. Estimated world requirements for 2017- statistics for 2015. Retrieved on January 28. 2019: https://www.incb.org/incb/en/narcoti drugs/Technical Reports/narcotic_drugs_reports.html

6. Procedure specific postoperative pain management. Retrieved on January 28, 2019 from URL: https://www.postoppain.org/

7. Chou R, Gordon DB, de Leon-Casasola OA, Rosenberg JM, Bickler S, Brennan $T$, et al. Management of Postoperative Pain: a clinical practice guideline from the American pain society, the American Society of Regional Anesthesia and Pain Medicine, and the American Society of Anesthesiologists' committee on regional anesthesia, executive committee, and administrative council. J Pain. 2016;17(2):131-57. https://doi.org/10.101 6/j.jpain.2015.12.008.

8. Practice Guidelines for Acute Pain Management in the Perioperative Setting An Updated Report by the American Society of Anesthesiologists Task Force on Acute Pain Management. Anesthesiol 2012; 116:248-273, 2, DOI: https://doi.org/10.1097/ALN.0b013e31823c1030.
9. Hjørland B. Classical databases and knowledge organization: a case for boolean retrieval and human decision-making during searches. J Assoc Informa Sci Tech. 2015;66(8):1559-75. https://doi.org/10.1002/asi.23250.

10. Stansfield C, Dickson K, Bangpan M. Exploring issues in the conduct of website searching and other online sources for systematic reviews: how can we be systematic? Systematic Reviews. 2016;5(1):191. https://doi.org/10.11 86/s13643-016-0371-9.

11. World Bank data on LMIC. Accessed on May 13, 2019 at URL: https://data. worldbank.org/income-level/lower-middle-income can J Anaesth 2016 Dec 1; 63(12):1335-1344.

12. Bhatia N, Arora S, Jyotsna W, Kaur G. Comparison of posterior and subcostal approaches to ultrasound-guided transverse abdominis plane block for postoperative analgesia in laparoscopic cholecystectomy. J Clin Anesth. 2014;26(4):294-9. https://doi.org/10.1016/j.jclinane.2013.11.023.

13. Shukla U, Prabhakar T, Malhotra K, Srivastava D, Malhotra K. Intraperitoneal bupivacaine alone or with dexmedetomidine or tramadol for post-operative analgesia following laparoscopic cholecystectomy: a comparative evaluation. Indian J Anaesth. 2015;59(4):234-9. https://doi.org/10.4103/00195049.155001.

14. Sinha S, Palta S, Saroa R, Prasad A. Comparison of ultrasound-guided transversus abdominis plane block with bupivacaine and ropivacaine as adjuncts for postoperative analgesia in laparoscopic cholecystectomies. Indian J Anaesth. 2016;60(4):264-9. https://doi.org/10.4103/0019-5049.179464.

15. Kamhawy G, El-Taher E, Abdelrahman M. A comparison of oblique subcostal transversus abdominis plane block versus thoracic paravertebral block for postoperative analgesia after open cholecystectomy. Egypt J Anaesth. 2017; 33(4):323-9. https://doi.org/10.1016/j.egja.2017.08.007.

16. Saxena R, Joshi S, Srivastava K, Tiwari S, Sharma N, Valecha UK. Comparative study of ultrasound-guided abdominal field blocks versus port infiltration in laparoscopic cholecystectomies for post-operative pain relief. Indian J Anaesth. 2016;60(8):578-83. https://doi.org/10.4103/0019-5049.187790.

17. Ali A, Babar KM. Comparison of preoperative dose of pregabalin with celecoxib for attenuation of postoperative pain after open cholecystectomy. Anaesth Pain Intensive Care. 2019:137-41.

18. Suseela I, Krishnadas Anandan AA, Kaniyil S. Comparison of ultrasoundguided bilateral subcostal transversus abdominis plane block and port-site infiltration with bupivacaine in laparoscopic cholecystectomy. Indian J Anaesth. 2018;62(7):497-501. https://doi.org/10.4103/ija.IJA_55_18.

19. Anand LK, Sandhu M, Singh J, Mitra S. Evaluation of analgesic efficacy of pregabalin for postoperative pain relief after laparoscopic cholecystectomy: a double blind study. APICARE. 2019:174-080.

20. Pasha AK, Farhat K, Ali S. effectiveness and safety of gabapentin in the management of post-operative pain. J Postgraduate Medical Institute (Peshawar-Pakistan). 2018;32(1):91-4.

21. Khan KK, Khan RI. Analgesic effect of bilateral subcostal tap block after laparoscopic cholecystectomy. J Ayub Med Coll Abbottabad. 2018;30:12-5

22. Jain S, Nazir N, Singh S, Sharma S. A prospective randomised controlled study for evaluation of high-volume low-concentration intraperitoneal bupivacaine for post-laparoscopic cholecystectomy analgesia. Indian J Anaesth. 2018;62(2):109-14. https://doi.org/10.4103/ija.IJA_87_17.

23. Amr YM, Yousef AA, Alzeftawy AE, Messbah WI, Saber AM. Effect of preincisional epidural fentanyl and bupivacaine on postthoracotomy pain and pulmonary function. Ann Thorac Surg. 2010;89(2):381-5. https://doi. org/10.1016/j.athoracsur.2009.10.060.

24. Khalil AE, Abdallah NM, Bashandy GM, Kaddah TA. Ultrasound-guided serratus anterior plane block versus thoracic epidural analgesia for thoracotomy pain. J Cardiothorac Vasc Anesth. 2017;31(1):152-8. https://doi. org/10.1053/j.jvca.2016.08.023.

25. Dutta V, Kumar B, Jayant A, Mishra AK. Effect of continuous paravertebral dexmedetomidine administration on intraoperative anesthetic drug requirement and post-thoracotomy pain syndrome after thoracotomy: a randomized controlled trial. J Cardiothorac Vasc Anesth. 2017;31(1):159-65.

26. Biswas S, Verma R, Bhatia VK, Chaudhary AK, Chandaa G, Prakash R. Comparison between thoracic epidural block and thoracic paravertebral block for post thoracotomy pain relief. J Clin Diagn Res. 2016;10(9):UC08-12. https://doi.org/10.7860/JCDR/2016/19159.8489.

27. Bakshi SG, Mapari A, Shylasree TS. REctus Sheath block for postoperative analgesia in gynecological ONcology Surgery (RESONS): arandomizedcontrolled trial. Can J Anesth. 2016;63(12):1335-44.

28. Dhanapal B, Sistla SC, Badhe AS, Ali SM, Ravichandran NT, Galidevara !. Effectiveness of continuous wound infusion of local anesthetics after 
abdominal surgeries. J Surg Res. 2017;212:94-100. https://doi.org/10.1016/j. jss.2016.12.027.

29. Wahba SS, Kamal SM. Analgesic efficacy and outcome of transversusabdominis plane block versus low thoracic-epidural analgesia after laparotomy in ischemic heart disease patients. J Anesth. 2014;28(4):517-23. https://doi.org/10.1007/s00540-013-1774-6.

30. Sethi M, Sethi N, Jain P, Sood J. Role of epidural ketamine for postoperative analgesia after upper abdominal surgery. Indian J Anaesth. 2011;55:141-5.

31. Moawad HE, Mokbel EM. Postoperative analgesia after major abdominal surgery: fentanyl-bupivacaine patient controlled epidural analgesia versus fentanyl patient controlled intravenous analgesia. Egypt J Anaesth. 2014; 30(4):393-7. https://doi.org/10.1016/j.egja.2014.06.002.

32. Patil SS, Kudalkar AG, Tendolkar BA. Comparison of continuous epidural infusion of $0.125 \%$ ropivacaine with $1 \mu \mathrm{g} / \mathrm{ml}$ fentanyl versus $0.125 \%$ bupivacaine with $1 \mu \mathrm{g} / \mathrm{ml}$ fentanyl for postoperative analgesia in major abdominal surgery. J Anaesthesiol Clin Pharmacol. 2018;34:29-34.

33. Bharti N, Pokale SN, Bala I, Gupta V. Analgesic efficacy of dexmedetomidine versus fentanyl as an adjunct to thoracic epidural in patients undergoing upper abdominal surgery: a randomized controlled trial. SAJAA. 2018;24:16-21.

34. Alvi $M$, Hussain $U$, Hussain $R$, Javaid $Y$, Nazeer T, Tahir A. Comparison of post-operative pain score with Transversus Abdominis plane (TAP) block versus placebo in open appendectomy. P J M H S. 2017;11:1121-3.

35. Patel I, Gandhi R, Shah A, Kumar P. Study to evaluate transversus abdominis plane (TAP) block with ropivacaine in appendectomy patients by total requirement of diclofenac as a postoperative analgesia drug. APICARE. 2019: 33-7.

36. Mishra A, Verma R, Bhatia VK, Chandra G, Gupta R, Chaudhary A. Thoracic Paravertebral Block for Postoperative Pain Management in Patients Undergoing for Nephrectomy: A Randomised Clinical Trial. J Clin Diagn Res. 2018:12(10):5-8.

37. Bharti N, Kumar P, Bala I, Gupta V. The efficacy of a novel approach to transversus abdominis plane block for postoperative analgesia after colorectal surgery. Anesth Analg. 2011;112(6):1504-8. https://doi.org/1 0.1213/ANE.0b013e3182159bf8.

38. Chotton T, Singh NR, Singh LC, Laithangbam PS, Singh HS. The effect of pregabalin for relief of postoperative pain after abdominal hysterectomy. Med Soc. 2014;28:18-21.

39. Badawy AA, El Sakka A. Preoperative gabapentin alone or in combination with dexamethasone on postoperative pain relief after abdominal hysterectomies. A randomized controlled trial. Egypt J Anaesth. 2015;31(2): 107-13. https://doi.org/10.1016/j.egja.2014.12.010.

40. Bashandy GM, Abbas DN. Pectoral nerves I and II blocks in multimodal analgesia for breast cancer surgery: a randomized clinical trial. Reg Anesth Pain Med. 2015:40:68-74

41. Hetta DF, Mohamed MA, Mohammad MF. Analgesic efficacy of pregabalin in acute postmastectomy pain: placebo controlled dose ranging study. J Clin Anesth. 2016;34:303-9. https://doi.org/10.1016/j.jclinane.2016.05.007.

42. Kasimahanti R, Arora S, Bhatia N, Singh G. Ultrasound-guided single-vs double-level thoracic paravertebral block for postoperative analgesia in total mastectomy with axillary clearance. J Clin Anesth. 2016;33:414-21. https:// doi.org/10.1016/j.jclinane.2016.01.027.

43. Kulhari S, Bharti N, Bala I, Arora S, Singh G. Efficacy of pectoral nerve block versus thoracic paravertebral block for postoperative analgesia after radical mastectomy: a randomized controlled trial. Br J Anaesth. 2016;117(3):382-6. https://doi.org/10.1093/bja/aew223.

44. Gupta K, Srikanth K, Girdhar KK, Chan V. Analgesic efficacy of ultrasoundguided paravertebral block versus serratus plane block for modified radical mastectomy: A randomised, controlled trial. Indian J Anaesth. 2017;61:381-6,

45. Bhuvaneswari V, Wig J, Mathew PJ, Singh G. Post-operative pain and analgesic requirements after paravertebral block for mastectomy: a randomized controlled trial of different concentrations of bupivacaine and fentanyl. Indian J Anaesth. 2012;56(1):34-9. https://doi.org/10.4103/0019-5049.93341.

46. Mahran E, Hassan ME. Comparison of pregabalin versus ketamine in postoperative pain management in breast cancer surgery. Saudi J Anaesth. 2015;9:253-7.

47. Kundra P, Varadharajan R, Yuvaraj K, Vinayagam S. Comparison of paravertebral and interpleural block in patients undergoing modified radical mastectomy. J Anaesthesiol Clin Pharmacol. 2013;29(4):459-64. https://doi. org/10.4103/0970-9185.119133.
48. Neethu M, Pandey RK, Sharma A, et al. Pectoral nerve blocks to improve analgesia after breast cancer surgery: a prospective, randomized and controlled trial. J Clin Anesth. 2018;45:12-7.

49. Mukherjee A, Das A, Mayur N, Bhattacharyya C, Biswas H, Mitra T, et al. Comparative evaluation of analgesic sparing efficacy between dexmedetomidine and clonidine used as adjuvant to ropivacaine in thoracic paravertebral block for patients undergoing breast cancer surgery: a prospective, randomized, double-blind study. Saudi J Anaesth. 2018;12(4): 548-54. https://doi.org/10.4103/sja.SJA_81_18.

50. Megha T, Hegde HV, Rao PR. Paravertebral block with morphine or dexmedetomidine as adjuvant to bupivacaine for post-operative analgesia in modified radical mastectomy: a prospective, randomised, double-blind study. Indian J Anaesth. 2018;62(6):424-30. https://doi.org/10.4103/ija.IJA_79_18.

51. Manzoor S, Taneja R, Sood N, Puri A, Kadayaprath G. Comparative study to assess the quality of analgesia of bupivacaine and bupivacaine with dexmedetomidine in ultrasound-guided pectoral nerve block type I and I| in breast surgeries. J Anaesthesiol Clin Pharmacol. 2018;34:227-31.

52. Kumar S, Goel D, Sharma SK, Ahmad S, Dwivedi P, Deo N, et al. A randomised controlled study of the post-operative analgesic efficacy of ultrasound-guided pectoral nerve block in the first 24 hafter modified radical mastectomy. Indian J Anaesth. 2018;62(6):436-42. https://doi.org/1 0.4103/ija.IJA_523_17.

53. Bharti N, Sardana DK, Bala I. The analgesic efficacy of dexmedetomidine as an adjunct to local anesthetics in supraclavicular brachial plexus block: a randomized controlled trial. Anesth Analg. 2015;121(6):1655-60. https://doi. org/10.1213/ANE.0000000000001006.

54. Kumar N, Thapa D, Gombar S, Ahuja V, Gupta R. Analgesic efficacy of preoperative stellate ganglion block on postoperative pain relief: a randomised controlled trial. Anaesthesia. 2014;69(9):954-660. https://doi.org/10.1111/ana e.12774.

55. Mullaji A, Kanna R, Shetty GM, Chavda V, Singh DP. Efficacy of periarticular injection of bupivacaine, fentanyl, and methylprednisolone in total knee arthroplasty: a prospective, randomized trial. J Arthroplasty. 2010;25:851-7.

56. Khanna A, Saxena R, Dutta A, Ganguly N, Sood J. Comparison of ropivacaine with and without fentanyl vs bupivacaine with fentanyl for postoperative epidural analgesia in bilateral total knee replacement surgery. J Clin Anesth. 2017;37:7-13. https://doi.org/10.1016/j.jclinane.2016.08.020.

57. Anis S, El Moaty NA, Youssef A, Ramzy R, Hassan R. Lumbar plexus block as a method of postoperative analgesia after hip surgery. Egypt J Anaesth. 2011;27(2):127-33. https://doi.org/10.1016/j.egja.2011.02.001.

58. Sawhney KY, Kundra S, Grewal A, Katyal S, Singh G, Kaur A. A randomized double blinded comparison of epidural infusion of bupivacaine, ropivacaine, bupivacaine-fentanyl, ropivacaine-fentanyl for postoperative pain relief in lower limb surgeries. J Clin Diagn Res. 2015;9(9):UC19-23. https://doi.org/10. 7860/JCDR/2015/15157.6459.

59. Trabelsi W, Gabsia AB, Lebbi A, Sammoud W, Labbène I, Ferjani M. Suprascapular block associated with supraclavicular block: an alternative to isolated interscalene block for analgesia in shoulder instability surgery? Orthop Traumatol Surg Res. 2017;103(1):77-83. https://doi.org/10.1016/j. otsr.2016.10.012.

60. Meghana S, Garima A, Madhavi S, Preet K, Karishma B. Efficacy of bupivacaine and ropivacaine for postoperative analgesia in continuous epidural infusion in lower limb surgeries under combined spinal-epidural analgesia. Anaesth Pain Intensive Care. 2019;20:360-5.

61. Thakur D, Malde A. Buprenorphine for postoperative analgesia: axillary brachial plexus block versus intramuscular administration in a placebocontrolled trial. J Anaesthesiol Clin Pharmacol. 2015;31(3):360-4. https://doi. org/10.4103/0970-9185.161673.

62. Mostafa SF, Eid GM, Elkalla RS. Patient-controlled fascia iliaca compartment block versus fentanyl patient-controlled intravenous analgesia in patients undergoing femur fracture surgery. Egy J Anaesth. 2018;34(1):9-13. https:// doi.org/10.1016/j.egja.2017.12.002.

63. PROSPECT. https://www.postoppain.org/sections/?root_id $=27323 \&$ section=4 Accessed 16 Jan 2019

64. Overall PROSPECT recommendations for laparoscopic cholecystectomy. Accessed 8 May 2019 at URL: https://archive.postoppain.org/sections/?root_ id $=40492$

65. A Mesbah, J Yeung, M. Pain after thoracotomy. BJA Education, 2016;16: 1-7, 1, DOI: https://doi.org/10.1093/bjaceaccp/mkv005. 
66. Azari L, Santoso JT, Osborne SE. Optimal pain management in total abdominal hysterectomy. Obst Gynecolo Survey. 2013;68(3):215-27. https:// doi.org/10.1097/OGX.0b013e31827f5119.

67. Hutchins JL. Improving patient outcomes through state-of-the-art pain control in Breast cancer surgery. Am J Hema/Oncol. 2015;11(5):29-32.

68. PROSPECT Non-cosmetic breast surgery subgroup recommendations. Accessed at URL: https://www.postoppain.org/sections/?root_id=4301 $0 \&$ section $=10$

69. PROSPECT overall recommendations for postoperative pain management following total knee arthroplasty: Accessed 4 Jan 2019 at URL: https://a rchive.postoppain.org/sections/?root_id=48292

\section{Publisher's Note}

Springer Nature remains neutral with regard to jurisdictional claims in published maps and institutional affiliations.

Ready to submit your research? Choose BMC and benefit from:

- fast, convenient online submission

- thorough peer review by experienced researchers in your field

- rapid publication on acceptance

- support for research data, including large and complex data types

- gold Open Access which fosters wider collaboration and increased citations

- maximum visibility for your research: over $100 \mathrm{M}$ website views per year

At BMC, research is always in progress.

Learn more biomedcentral.com/submissions 